\title{
O programa de aprimoramento profissional em psicologia na perspectiva do egresso
}

\author{
Evelyn Francine Dutra Pereira* \\ Flavia Helena Pereira Padovani**
}

Resumo

Os Programas de Aprimoramento Profissional foram criados na década de setenta e tem seu valor reconhecido como formação de pós-graduação na modalidade latu sensu dentro do território do estado de São Paulo. O presente estudo teve por objetivo identificar o impacto dos Programas de Aprimoramento Profissional na formação e exercício profissional do psicólogo, especialmente na área da saúde, de acordo com a percepção dos egressos. Trata-se de um estudo transversal, descritivo, de abordagem quanti-qualitativa. Grande parte dos egressos $(93,1 \%)$ encontra-se inserida no mercado de trabalho, atuando como psicólogo. As atividades práticas foram melhores avaliadas do que as atividades teóricas. Observou-se que a realização do Programa de Aprimoramento Profissional possibilitou o aperfeiçoamento e a capacitação do profissional para atuação qualificada e diferenciada na área da saúde. Por fim, cabe ressaltar, a necessidade de adequação dos Programas de Aprimoramento às novas demandas em saúde.

Palavras-chaves: Psicologia da saúde; Formação profissional; Ensino Superior; Pós-graduação.

\section{The professional improvement programs in psychology in the perspective of the egress}

\begin{abstract}
Professional Enhancement Programs were created in the 1970s and had the post-graduate training in the lato sensu modality within the territory of the state of São Paulo. The present study aimed to identify the impact of the learning programs in the health and exercise area of the psychologist, especially in the health area, according to the perception of the graduates. This is a cross-sectional, descriptive, quantitative-qualitative study. Most of the graduates (93.1\%) are in the job market, working as a psychologist. The practical activities were better evaluated than the theoretical activities. It was observed that the realization of the Professional Improvement Program enables the improvement and the qualification of the professional to perform qualified and differentiated in the health area. Finally, it is worth mentioning the need to adapt the Enhancement Programs to the new health demands.
\end{abstract}

Key-Words: Health psychology; Professional education; Higher education; Postgraduate training.

* Psicóloga pela Universidade do Sagrado Coração.

** Psicóloga e Doutora pela FCLRP-USP. Professora Assistente do Depto de Neurologia, Psicologia e Psiquiatria da FMB-UNESP. 
Desde a regulamentação da psicologia como profissão no Brasil, em 27 de agosto de 1962, a psicologia clínica, como atividade autônoma realizada em ambiente de consultório, tem sido a atividade profissional dominante, com maior concentração de profissionais (Krug, Bastianello, Port \& Hutz, 2012; Martins, Matos \& Maciel, 2009; Pereira \& Pereira Neto, 2003).

Contudo, o mercado de trabalho na área clínica se encontra saturado e, dessa forma, outras áreas de atuação vêm se expandindo, como a psicologia da saúde (Bardagi, Bizarro, Andrade, Audibert \& Lassance, 2008; Castro \& Bornholdt, 2004; Krug, Bastianello, Port \& Hutz, 2012; Martins, Matos \& Maciel, 2009; Pereira \& Pereira Neto, 2003). Gorayeb, Borges e Oliveira (2012) destacam que a entrada de forma mais expressiva dos profissionais de psicologia nos serviços de saúde em nosso país ocorreu a partir da década de oitenta. Segundo os autores, a grave crise econômica dessa época, culminou em dificuldades de grande parte dos profissionais em realizarem a manutenção de suas clínicas particulares, favorecendo a procura dos profissionais por outros campos de atuação.

Após o período inicial de Psicologia da Saúde com a inserção do psicólogo nas instituições hospitalares, os movimentos de reorganização do sistema público de saúde, com a implementação de programas com ênfase na área ambulatorial e incentivo na formação de equipes multiprofissionais, além das modificações na área de saúde mental, decorrentes da reforma psiquiátrica brasileira, que focava a substituição dos hospitais psiquiátricos pelo cuidado em serviços de atenção psicossocial, contribuíram para a expansão da atuação do psicólogo na saúde (Gorayeb, Borges \& Oliveira (2012).

Mais recentemente, com a implementação do Sistema Único de Saúde (SUS), estabelecido formalmente a partir da Constituição Federal de 1988, e a regulamentação dos deveres das diferentes esferas governamentais para garantir tais direitos à população (Mercadante, Schechtman, Cortes, Munhoz Junior, Mendes, Un, Medeiros, Lemos, Serrano, Meirelles, Nogueira, Piola, Vianna \& Figueiredo, 2002)foram observadas novas possibilidades de inserção e novas demandas aos profissionais que atuam na área da saúde.

Houve, consequentemente, uma necessidade de investimentos na formação teórica e técnica, considerando que a maioria dos profissionais psicólogos não está preparada para o trabalho fora dos contextos tradicionais (Bardagi, Bizarro, Andrade, Audibert \& Lassance, 2008). De um modo geral, profissionais que atuam na área da saúde, incluindo a psicologia hospitalar, muitas vezes, tendem a desenvolver atividades profissionais baseadas no modelo clínico, com predomínio de intervenção psicoterápica individual e/ou de grupo (Seidl \& Costa Jr, 1999).

Entretanto, a atuação do psicólogo na área da saúde tem especificidades. A transposição das orientações teóricas e práticas, embasadas em atividades individualizadas, mantendo atendimentos contínuos e prolongados, realizados no exercício clínico tradicional, para os serviços públicos de saúde, inviabilizam práticas profissionais que garantam o bem-estar da população (Böing \& Crepaldi, 2014; Gorayeb 2010; Gorayeb, Borges \& Oliveira, 2012; Pitombeira, Barroso, Xavier \& Oliveira, 2016).

A formação do psicólogo para atuar nessa área da saúde deveria contemplar, entre outros aspectos, conhecimentos sobre: bases biológicas, sociais e psicológicas da saúde e da doença; avaliação, assessoramento e intervenção em saúde; políticas e organização de saúde e colaboração interdisciplinar; temas profissionais, éticos e legais; e conhecimentos de metodologia e pesquisa em saúde (Castro \& Bornholdt, 2004). Pitombeira, Barroso, Xavier \& Oliveira (2016) enfatizam que a atuação do psicólogo no campo da saúde, principalmente no da saúde pública, deve contemplar uma discussão da atenção à saúde de forma ampla, de modo a fortalecer o SUS, reforçando seus princípios doutrinários.

Os Programas de Aprimoramento Profissionais (PAP) do Estado de São Paulo, criados em 11/09/1979 pelo Decreto Estadual n ${ }^{\circ} 13.919$, visam capacitar os profissionais não médicos, prioritariamente os recém-graduados, ao exercício profissional, treinando-os em serviço supervisionado por profissionais qualificados, em atividades desenvolvidas em instituições vinculadas ao Sistema Único de Saúde (SUS) (Secretaria de Saúde Estado de São Paulo, 2016).

Verifica-se que, embora haja especificidades em cada programa, todos possuem como objetivo comum capacitar os profissionais dessas áreas a desempenharem seus trabalhos de forma crítica e diferenciada, de acordo com os princípios e diretrizes do SUS, abarcando os diversos níveis de abrangência à saúde (primário, secundário e terciário) de forma a orientarem suas ações para a melhoria de condições de saúde a população (Secretaria de Saúde do Estado de São Paulo, 2016). Tais programas possuem duração de um a dois anos de formação, com carga horária semanal de 40 horas. As atividades práticas realizadas constituem oitenta por cento dos programas, enquanto que a parte teórica, distribuídas em aulas, seminários, desenvolvimento das monografias, entre outras modalidades pedagógicas, totalizam os restantes vinte por 
cento. Deste modo, obtém-se uma carga horária anual de atividades de, no mínimo, mil setecentas e sessenta horas (http://fmb.unesp.br/).

No âmbito Faculdade de Medicina de Botucatu (FMB), da Universidade Júlio Mesquita Filho (UNESP), os Programas de Aprimoramento Profissional tiveram início no ano de 1981. Atualmente, existem 24 programas oferecidos pela FMB-UNESP que abarcam profissionais de diversas áreas. Entre os programas oferecidos pela FMB-UNESP, cinco são destinados à formação e capacitação do profissional de psicologia, sendo esses: psicologia da saúde, psicologia em diálise, psicologia em saúde coletiva, psicologia em gastroenterologia e psicologia hospitalar em pediatria (http:// fmb.unesp.br/).

O presente estudo teve por objetivo identificar o impacto dos Programas de Aprimoramento Profissional na formação e exercício profissional do psicólogo, especialmente na área da saúde, de acordo com a percepção dos egressos.

\section{Material e método}

Trata-se de um estudo transversal, descritivo, de abordagem quanti-qualitativa.

\section{Aspectos Éticos}

Este trabalho foi submetido e aprovado pelo Comitê de Ética em Pesquisa (CEP) da Faculdade de Medicina de Botucatu (FMB) - UNESP (CAAE 58949016.5.0000.5411), seguindo as recomendações do Comitê Nacional de Ética Pesquisa (CONEP) e em concordância com as diretrizes vigentes.

\section{Participantes}

Trata-se de amostra de conveniência, formada por 29 (vinte e nove) psicólogos, de ambos os sexos, egressos dos Programas de Aprimoramento Profissional (PAP) em psicologia da Faculdade de Medicina de Botucatu UNESP, tendo concluído o programa entre os anos de 1982 até o primeiro semestre de 2016.

Ao todo, foram contatados 50 (cinquenta) egressos, sendo que 21 (vinte e um) não concordaram em participar ou não responderam os questionários.

\section{Local e Contexto da Pesquisa}

Conforme citado anteriormente, os Programas de Aprimoramento Profissional (PAP) da Faculdade de Medicina de Botucatu (FMB), da Universidade Júlio Mesquita Filho (UNESP), foram criados no ano de 1981.
Atualmente, são oferecidas seis vagas aos profissionais de psicologia em cinco programas da FMB-UNESP: psicologia da saúde (2), psicologia em diálise (1), psicologia em saúde coletiva (1), psicologia em gastroenterologia (1) e psicologia hospitalar em pediatria (1) (http://fmb. unesp.br/).

\section{Instrumentos}

Para a realização do presente estudo, foi elaborado um questionário semiestruturado com 15 questões, a fim de coletar dados sobre a graduação do participante, do Programa de Aprimoramento Profissional (PAP) realizado, da atuação profissional atual e sua opinião sobre a interface entre o PAP desenvolvido e sua formação e atuação profissional.

\section{Procedimento}

\section{Coleta de dados}

Os participantes elegíveis para o presente estudo foram identificados através de indicações do setor responsável pelos Programas de Aprimoramento Profissional (PAP) da FMB - UNESP, além de consultas aos preceptores responsáveis pelos diversos Programas (PAP) em psicologia, assim como o contato com os atuais aprimorandos desses e outros egressos.

A partir dessas indicações, os participantes foram contatados pessoalmente ou através de telefonemas e mensagens de e-mail, sendo convidados a participar do estudo. Foram, então, enviados por e-mail ou entregues pessoalmente, o Termo do Consentimento Livre e Esclarecido (TCLE) e o questionário a ser preenchido.

\section{Análise de dados}

Primeiramente, foi realizada a preparação dos dados quantitativos com a elaboração de um banco de dados. Em seguida, realizou-se a análise descritiva dos dados, em termos de frequências absoluta e relativa.

Os dados obtidos através das questões abertas foram submetidos à análise de conteúdo, a qual consiste em estudar o assunto latente das mensagens emitidas, organizando, primeiramente, os resultados obtidos com a finalidade de fazer uma leitura flutuante (permitir que todos os pensamentos com relação às mensagens sejam consideráveis no primeiro momento da análise), para depois explorar o material fazendo hipóteses referentes a esse, que foram interpretadas (decodificadas) de acordo com o objetivo do trabalho (Bardin, 1991). 


\section{Resultados}

De acordo com os resultados apresentados os egressos dos Programas de Aprimoramento Profissional em Psicologia da Faculdade de Medicina de Botucatu UNESP que participaram dessa pesquisa concluíram a graduação a partir de 2001 (86,2\%) e, consequentemente, participaram do Programa de Aprimoramento Profissional (PAP) entre os anos de 2010 e 2015 (65,5\%). A graduação em psicologia foi realizada majoritariamente no estado de São Paulo (96,5\%), em instituição pública de ensino superior $(69,0 \%)$. Pouco mais da metade dos participantes era egresso dos programas (PAP) de Psicologia da Saúde $(38,0 \%)$ ou Psicologia Hospitalar em Pediatria (20,7\%).

Tabela 1: Dados de formação da Graduação e do PAP do egresso

\begin{tabular}{lcc}
\hline Graduação & $\mathrm{n}$ & $\%$ \\
\hline Graduação & $\mathrm{n}$ & $\%$ \\
Ano de formação & & \\
$\quad 1980$ a 1990 & 01 & $3,5 \%$ \\
1991 a 2000 & 03 & $10,3 \%$ \\
$\quad 2001$ em diante & 25 & $86,2 \%$ \\
Estado de formação & & \\
$\quad$ São Paulo & 28 & $96,5 \%$ \\
$\quad$ Outro & 01 & $3,5 \%$ \\
Tipo de Instituição & & \\
$\quad$ Pública & 20 & $69,0 \%$ \\
$\quad$ Privada & 09 & $31,0 \%$ \\
\hline Programa de Aprimoramento & $\mathrm{n}$ & $\%$ \\
Profissional (PAP) & & \\
\hline Programa PAP & & \\
$\quad$ Psicologia da Saúde & 11 & $38,0 \%$ \\
Psicologia Hospitalar em Pediatria & 6 & $20,7 \%$ \\
Psicologia em Diálise & 5 & $17,2 \%$ \\
$\quad$ Psicologia em Gastroenterologia & 4 & $13,8 \%$ \\
Psicologia em Saúde Pública & 3 & $10,3 \%$ \\
Período de formação no PAP & & \\
1980 a 2000 & 4 & $13,8 \%$ \\
$\quad 2000$ a 2010 & 6 & $20,7 \%$ \\
$\quad 2010$ a 2015 & 19 & $65,5 \%$ \\
\hline
\end{tabular}

Todos os egressos participantes do estudo apontaram que estavam satisfeitos, plena ou parcialmente, com as atividades desenvolvidas. Entretanto, observa-se que as atividades práticas foram melhor avaliadas, já que $82,7 \%$ se mostraram plenamente satisfeitos, enquanto $55,2 \%$ estavam plenamente satisfeitos com as atividades teóricas.
Tabela 2: Avaliação do Egresso Sobre o PAP Realizado

\begin{tabular}{lcc}
\hline $\begin{array}{l}\text { Qualidade do Programa de Aprimo- } \\
\text { ramento Profissional (PAP) }\end{array}$ & n & $\%$ \\
\hline Atividades Teóricas & & \\
$\quad$ Plenamente satisfatórias & 16 & $55,2 \%$ \\
$\quad$ Parcialmente satisfatórias & 13 & $44,8 \%$ \\
$\quad$ Insatisfatórias & 00 & - \\
\hline Atividades Práticas & & \\
$\quad$ Plenamente satisfatórias & 24 & $82,7 \%$ \\
$\quad$ Parcialmente satisfatórias & 5 & $17,3 \%$ \\
$\quad$ Insatisfatórias & 00 & - \\
\hline
\end{tabular}

De acordo com as respostas às perguntas abertas, pode-se notar que aqueles estavam plenamente satisfeitos com as atividades práticas realizadas no programa de Aprimoramento Profissional (PAP) apontaram os seguintes aspectos positivos: o desenvolvimento das habilidades profissionais, integração entre teoria e prática, preparação para o mercado de trabalho, contato com a atuação multidisciplinar e ampliação da prática após a graduação. Tais aspectos podem ser exemplificados na avaliação de um dos egressos:

"A parte prática foi um dos melhores desafios vivenciados, pois além de colocar em prática todo conhecimento adquirido na graduação, foi possivel desenvolver habilidades e competências de modo a se tornar apto para a atuação posterior ao programa. Sem dúvida, é um programa enriquecedor de modo que proporciona conbecimentos acerca da psicologia, principalmente a área de saúde mental que foi de minha atuação" (Psicólogo 11).

Por outro lado, o contato limitado com a rede de serviço de saúde foi citado como um aspecto a ser melhorado por aqueles que estavam parcialmente satisfeitos com as atividades práticas do Programa de Aprimoramento Profissional (PAP).

Em relação à parte teórica do PAP, o aspecto positivamente avaliado por aqueles que se encontravam plenamente satisfeitos foi, novamente: a integração do conteúdo teórico abordado com a prática realizada no PAP. Segundo a percepção dos egressos as atividades como supervisões, aulas, seminários e discussões clínicas desenvolvidas serviam como preparação dos psicólogos aprimorandos na execução prática das atividades. Por outro lado, aqueles que se encontravam parcialmente 
satisfeitos referiram que os conteúdos foram administrados de forma superficial e pouco abrangente, principalmente aqueles relativos à área da saúde pública e atenção primária.

Cabe ressaltar que, como nem todos os egressos participantes encontravam-se inseridos no mercado de trabalho e aqueles que se encontravam, muitas vezes, estavam exercendo funções diferentes, o número de respostas a cada item variou.

Tabela 3: Exercício profissional do egresso

\begin{tabular}{lcc}
\hline & $\mathrm{n}$ & $\%$ \\
\hline $\begin{array}{l}\text { Atuação profissional como psicólogo } \\
(\mathrm{n}=29)\end{array}$ & \\
$\quad$ Sim & 27 & $93,1 \%$ \\
$\quad$ Não & 2 & $6,9 \%$ \\
Atuação em diferentes áreas (n= 27) & & \\
$\quad$ Sim & 17 & $63,0 \%$ \\
$\quad$ Não & 10 & $37,0 \%$ \\
Área de atuação (n=27) & & \\
$\quad$ Saúde & 18 & $66,7 \%$ \\
$\quad$ Clínica & 17 & $63,0 \%$ \\
$\quad$ Docência & 03 & $11,1 \%$ \\
$\quad$ Outra & 07 & $25,9 \%$ \\
Setor (n=27) & & \\
$\quad$ SUS & 18 & $66,7 \%$ \\
$\quad$ Público & 04 & $14,8 \%$ \\
Privado & 20 & $74,1 \%$ \\
$\quad$ Outro & & \\
& 02 & $7,4 \%$
\end{tabular}

Inserção no mercado de trabalho após o PAP $(n=28)$

Até 2 anos

De 2 a 5 anos

$27 \quad 96,4 \%$

$01 \quad 3,6 \%$

Dificuldade de inserção no mercado de trabalho $(n=28)$

Sim $05 \quad 17,9 \%$

Não $23 \quad 82,1 \%$

Grande parte dos egressos participantes (93,1\%) encontra-se inserida no mercado de trabalho, atuando como psicólogo. A maioria atua em diferentes áreas $(63,0 \%)$, especialmente nas áreas da saúde $(66,7 \%)$ e clínica $(63,0 \%)$. Porém, alguns egressos encontram-se em outras áreas como a docência, assistência social, psicologia jurídica, psicologia organizacional. O setor privado $(74,1 \%)$ e o Sistema Único de Saúde (SUS) (66,7\%) são os setores que mais absorveram os egressos do PAP. A maior parte dos egressos não encontrou dificuldade de inserção no mercado de trabalho $(82,1 \%)$, tendo iniciado suas atividades profissionais como psicólogo num prazo de até 2 anos (96,4\%) após o término do PAP.
Os egressos que responderam que não obtiveram dificuldades na inserção do mercado de trabalho, apontaram como aspectos facilitadores a possibilidade de escolha em relação a área de atuação profissional, aquisição de segurança para exercício da profissão como psicólogo, preparação teórica e técnica para o mercado de trabalho, valorização curricular após realização do Programa de Aprimoramento Profissional (PAP) e abertura de possibilidades de atuação após realização do programa. Por outro lado, alguns egressos relataram dificuldade em manter o exercício profissional na área clínica, bem como dificuldade em ingressar na área de interesse.

Complementarmente aos dados apresentados na Tabela 3, observa-se que os egressos foram unânimes em avaliar que a realização do Programa de Aprimoramento Profissional (PAP) influenciou sua inserção no mercado de trabalho. De acordo com a análise das perguntas abertas, tanto as atividades teóricas quanto as atividades práticas foram fundamentais para sua inserção no mercado de trabalho. Em relação às atividades teóricas, os egressos apontaram: a possibilidade de reflexão em relação a teoria e prática do psicólogo, possibilidade de aprofundar conteúdos teóricos e técnicos, principalmente em relação ao Sistema Único de Saúde (SUS) e psicologia hospitalar, o contato com o trabalho multidisciplinar, a ampliação da experiência obtida na graduação e a preparação do egresso para o mercado de trabalho.

Em relação à influência das atividades práticas do PAP para a inserção no mercado de trabalho, os egressos avaliaram positivamente: desenvolvimento das habilidades e competências, possibilidade de contato com vários níveis de atenção, profissionais e áreas de atuação da saúde, auxílio na escolha da área atual de atuação profissional, ampliação da experiência em diferentes possibilidades de atuação do psicólogo e amadurecimento profissional.

\section{Discussão}

O perfil do egresso participante da pesquisa, recém-graduado e proveniente de instituição de ensino superior do Estado de São Paulo, condiz com o perfil esperado. Conforme salientado anteriormente, o Programa de Aprimoramento Profissional (PAP) consiste em uma modalidade de formação/capacitação profissional na área da saúde, destinada a profissionais recém-graduados, sem vínculo empregatício (http://pap-saude.net.br/).

Cabe ressaltar ainda que, independentemente de o PAP ser um programa exclusivo de São Paulo, há uma maior concentração de cursos de graduação em psicologia no estado. Segundo um levantamento realizado por Lis- 
boa e Barbosa (2009), dos 396 cursos de graduação em psicologia cadastrados no Ministério da Educação (MEC) entre os anos de 2007 e 2008, a maioria deles se encontrava na Região Sudeste. O Estado de São Paulo sozinho era responsável por $26,26 \%$ do total dos cursos de graduação.

O Estado de São Paulo também concentra o maior número de psicólogos com registro ativo no Conselho Federal de Psicologia (CFP, 2016), proporcionando melhor distribuição dos profissionais ao longo do território nacional (Borges-Andrade, Bastos, Andery, Guzzo \& Trindade, 2015).

Por outro lado, apesar dos cursos de graduação em psicologia serem majoritariamente oferecidos em instituições privadas (Lisboa \& Barbosa, 2009), 69\% dos egressos participantes cursaram psicologia em instituições públicas. Mesmo que em menor número, as instituições públicas são sistematicamente melhor avaliadas que as instituições privadas no ENADE (Lisboa \& Barbosa, 2009), contam com um percentual maior de docentes titulados (Yamamoto, 2006) e concentram as atividades de pesquisa do país (Lisboa \& Barbosa, 2009).

Ainda em relação ao perfil do egresso, devido ao número de vagas oferecidas pelos Programas de Aprimoramento Profissional em psicologia da FMB - UNESP, ao longo dos anos (http://fmb.unesp.br/), a maioria dos egressos que participaram do trabalho havia concluído os programas (PAP) de Psicologia da Saúde $(38,0 \%)$ ou Psicologia Hospitalar em Pediatria (20,7\%).

Conforme esperado, os participantes desse trabalho eram, em sua maioria, aqueles que concluíram mais recentemente os Programas de Aprimoramento Profissional em Psicologia (PAP). Trata-se de uma amostra de conveniência, tendo sido contatados todos aqueles egressos identificados pelo setor responsável pelo PAP da FMB - UNESP, ou, principalmente, por meio de indicação dos preceptores responsáveis, dos atuais aprimorandos e de outros egressos. Portanto, participaram do estudo os egressos que, de alguma forma, mantinham dados de contato (e-mail, telefone, celular) atualizados com seus preceptores, colegas e ex-colegas. Assim, trata-se de uma limitação do presente estudo, indicando que os resultados devem ser considerados com parcimônia.

Considerando o objetivo do trabalho, de identificar o impacto dos Programas de Aprimoramento Profissional (PAP) em psicologia da FMB - UNESP, os resultados indicam um impacto positivo na formação e prática do exercício profissional do psicólogo.

Grande parte dos egressos participantes (93,1\%) encontrava-se inserida no mercado de trabalho, atuando como psicólogos. Este percentual é maior do que o encontrado por outros estudos com egressos de cursos de graduação em psicologia, tanto de universidade privada (Martins, Matos \& Maciel, 2009) como pública (Bardagi, Bizarro, Andrade, Audibert \& Lassance, 2008). Além disso, segundo a percepção dos egressos, sua inserção do mercado de trabalho foi rápida, em até dois anos $(96,4 \%)$ e sem dificuldades $(82,1 \%)$. Entre os aspectos identificados como aspectos facilitadores para a rápida inserção no mercado de trabalho, os egressos apontaram a preparação teórica e técnica proporcionada pelo PAP, fazendo com que os egressos se sentissem mais seguros para o exercício da profissão como psicólogo. Além disso, houve valorização curricular após realização do Programa de Aprimoramento Profissional (PAP).

Conforme destacado anteriormente, os Programas de Aprimoramento Profissional (PAP) têm como um de seus objetivos capacitar o aprimorando para atuação qualificada e diferenciada na área da saúde, promovendo o aperfeiçoamento do desempenho profissional nas diversas áreas que integram, apoiam ou gerenciam a prestação de serviços de saúde (SES/SP, 2016). Pode-se dizer que tal objetivo vem sendo atendido na medida em que a maioria dos egressos respondentes atua na área de saúde $(66,7 \%)$, especificamente e serviços no SUS (66,7\%).

Entretanto, assim como em outros estudos (Martins, Matos \& Maciel, 2009; Seidl \& Costa Jr., 1999), os psicólogos egressos atuam em mais de uma área (63\%), sendo a área clínica (63\%), no setor privado, a alternativa escolhida. Borges-Andrade, Bastos, Andery, Guzzo \& Trindade (2015), verificam que apesar da opção dos profissionais psicólogos por atuar na área da saúde, a remuneração é pouco considerada. Possivelmente, a baixa remuneração dos egressos que atuam na área fez com que eles buscassem outra atividade profissional concomitante. Macedo e Dimenstein (2012), em estudo com psicólogos que atuam em unidades básicas de saúde (UBS) apontam que estes profissionais estavam insatisfeitos com a remuneração oferecida, de três e quatro salários mínimos. Mesmo a jornada de trabalhado no setor de saúde público sendo de trinta horas semanais, os psicólogos complementavam sua renda com um segundo local de trabalho, com atuação em clínicas privadas, na rede hospitalar privada e na docência do ensino superior (Macedo \& Dimenstein, 2012), assim como os participantes do presente estudo.

A preferência pela clínica, como exercício profissional complementar à atuação da área da saúde, possivelmente, se deve ao fato de ainda ser o modelo predominante na formação do psicólogo brasileiro (Bardagi, 
Bizarro, Andrade, Audibert \& Lassance, 2008; Oliveira, Dantas, Costa, Silva, Alverga, Carvalho \& Yamamoto 2004), além da maior flexibilidade de horários e maior autonomia do profissional. Segundo Borges-Andrade et al. (2015), a principal porta de entrada no mercado de trabalho é o trabalho autônomo.

No geral, os egressos participantes se mostraram satisfeitos, plena ou parcialmente com o Programa de Aprimoramento Profissional (PAP) realizado e foram unânimes em avaliar que sua inserção no mercado de trabalho foi influenciada pela realização do PAP.

Entretanto, as atividades práticas foram melhor avaliadas do que as atividades teóricas. Segundo os egressos, as atividades práticas, possibilitaram a integração entre teoria e prática e ampliaram as vivências profissionais, especialmente no contexto da saúde e em equipe multiprofissional. Por se tratar de um programa de treinamento em serviço, com supervisão qualificada, as atividades práticas constituem $80 \%$ (oitenta por cento) das atividades desenvolvidas durante o PAP (SES/SP, 2016). Os currículos de Psicologia no nosso país contemplam, em grande parte, as atividades teóricas, (Lisboa \& Barbosa, 2009; Krug, Bastianello, Port \& Hutz, 2012). Ao possibilitar o desenvolvimento de habilidades e competências, e o amadurecimento profissional, as atividades práticas dos programas de PAP auxiliaram na escolha da área atual de atuação profissional e na inserção no mercado de trabalho.

Mesmo que de forma menos enfática, no geral, as atividades teóricas também foram bem avaliadas pelos egressos, auxiliando na preparação dos psicólogos aprimorandos na execução prática das atividades. Ao mesmo tempo em que conteúdos relacionados ao Sistema Único de Saúde (SUS) e à psicologia hospitalar foram destacados pelos egressos, tais profissionais, ainda apontam a necessidade de aprofundarem os embasamentos teóricos que permeiam as políticas de saúde, de saúde pública e a complexidade desse sistema. O contato limitado com a rede de serviço de saúde também foi citado como um aspecto a ser melhorado nas atividades práticas, denotando, assim, a necessidade de adequação dos programas de PAP às novas demandas em saúde, em decorrência especialmente da criação do Sistema Único de Saúde (SUS). Reforçando a necessidade de aprofundamento sobre as políticas de saúde, Pitombeira, Barroso, Xavier \& Oliveira (2016) enfatizam a discussão da atenção à saúde de forma ampla, de modo a fortalecer o SUS, reforçando seus princípios doutrinários.

As teorias psicológicas que norteiam as práticas do psicólogo dentro das políticas de saúde também foram mencionadas enquanto conteúdos a serem abordados de forma mais aprofundada, considerando que o modelo clínico tradicional não deve ser transferido para a área da saúde (Böing \& Crepaldi, 2014; Gorayeb, 2010; Gorayeb, Borges \& Oliveira, 2012; Pitombeira, Barroso, Xavier \& Oliveira (2016). Segundo Castro e Bornholdt (2004), a formação do psicólogo da saúde deveria contemplar, entre outros aspectos, conhecimentos sobre: bases biológicas, sociais e psicológicas da saúde e da doença; avaliação, assessoramento e intervenção em saúde; políticas e organização de saúde e colaboração interdisciplinar; temas profissionais, éticos e legais; e conhecimentos de metodologia e pesquisa em saúde.

Conclui-se com o presente estudo, que o perfil do egresso dessa pesquisa é, principalmente, daqueles que realizaram o Programa de Aprimoramento Profissional (PAP) em psicologia, a partir dos anos 2001 (dois mil e um), período em que as políticas de saúde, saúde pública e saúde mental passaram a se consolidar no país, após a reforma no sistema de saúde no final da década de oitenta. Grande parte dos egressos está trabalhando como psicólogo, prioritariamente na área da saúde, no SUS, reforçando, assim, o objetivo do PAP de capacitar os profissionais não médicos, prioritariamente os recém-graduados, ao exercício profissional, treinando-os em serviço supervisionado por profissionais qualificados, em instituições vinculadas ao Sistema Único de Saúde (SUS).

Os egressos avaliaram positivamente o impacto a realização do Programa de Aprimoramento Profissional (PAP) na sua formação e prática do exercício profissional apontando para o déficit que ainda necessita ser suprido em sua formação teórica, principalmente enquanto profissional da área da saúde. Por se tratar de um programa de treinamento em serviço, as atividades práticas são majoritárias, possibilitando o desenvolvimento de habilidades e competências, e o amadurecimento profissional. Porém, verifica-se a necessidade de adequação dos programas de PAP às novas demandas em saúde, em decorrência especialmente da criação do Sistema Único de Saúde (SUS).

Cabe ressaltar, entretanto, que por tratar-se de uma amostra de conveniência, os resultados precisam ser relativizados. Não foi possível o contato com a maior parte dos egressos que realizaram o PAP nas décadas de oitenta e noventa, do século passado, período em que as políticas de saúde, saúde pública e saúde mental ainda estavam se consolidando no país, após a reforma no sistema de saúde no final da década de oitenta, e a formação e preparação do profissional de psicologia possuía ênfase ainda mais significativa nas áreas clínicas e privadas. Sugere-se, dessa 
forma, que em investigações futuras sejam comparados os perfis e respostas de egressos, diferenciando-os quanto ao período de conclusão do PAP, buscando identificar o impacto das políticas públicas de saúde na formação dos profissionais psicólogos.

\section{Referências bibliográficas}

Andrade, J. F. S. de M. \& Simon, C. P. (2009). Psicologia na Atenção Primária à Saúde: reflexões e implicações práticas. Paidéia, 19 (43), 167-175.

Assembleia Legislativa do Estado de São Paulo. (1979, 11 de setembro). Recuperado em 07 de junho de 2016, de http://www.al.sp.gov.br/repositorio/ legislacao/decreto/1979.

Audibert, A. \& Lassance, M. C. P. (2008). Avaliação da formação e trajetória profissional na perspectiva de egressos de um curso de psicologia. Psicologia: Ciência e Profissão, 28 (2), 304-315.

Bardagi, M. P., Bizzaro, L., Andrade, A. M. J. de,

Bardin, L. (1991). Análise de conteúdo. Rio de Janeiro, RJ: Edições 70.

Böing, E. \& Crepaldi, M. A. (2014). Reflexões epistemológicas sobre o SUS e atuação do psicólogo. Psicologia: Ciência e Profissão, 34 (3), 745-760.

Borges-Andrade, J. E., Bastos, A. V. B., Andery, M. A. P. A., Guzzo, R. S. L. \& Trindade, Z. A. (2015). Psicologia brasileira: uma análise de seu desenvolvimento. Universitas Psychologica, 14 (3), 865-880.

Castro, E. K. de \& Bornholht, E. (2004). Psicologia da saúde $x$ Psicologia hospitalar: definições e possibilidades de inserção profissional. Psicologia: Ciência e Profissão, 24 (3), 48-57.

Conselho Federal De Psicologia. (2016). A Psicologia Brasileira Apresentada em Números [Versão Digital]. Recuperado de: http://www.cfp.org.br/infografico/quantos-somos/.

Conselho Regional De Psicologia. (2012). O avanço da profissão: um pouco de história. [Versão Digital]. Recuperado de: http://www.crpsp.org.br/portal/ comunicacao/jornal crp/172/frames/fr avancos.aspx.

Faculdade De Medicina De Botucatu (2016). Programa de Aprimoramento Profissional 2016-2017. Recuperado de: http://Fmb.unesp.br/\#!/aprimoramento.

Gorayeb, R. (2010). Psicologia da saúde no Brasil. Psicologia: Teoria e Pesquisa, 26, 115-122.

Gorayeb, R, Borges, C. D., \& Oliveira, C. M. de. (2012). Psicologia na Atenção Primária: ações e reflexões em programa de aprimoramento profissional. Psicologia: Ciência e Profissão, 32(3), 674-685.
Krug, J. S., Bastianello, M. R., Port, A. K. \& Hutz, C. S. (2013). Percepções de estudantes de psicologia sobre o domínio de teorias, áreas de exercício e atividades profissionais. Psicologia: Ensino \& Formação, 4 (1), 41-59.

Lisboa, F. S. \& Barbosa, A. J. G. (2009). Formação em psicologia no Brasil: um perfil dos cursos de graduação. Psicologia: Ciência e Profissão, 29 (4), 718-737.

Macedo, J. P. \& Dimenstein, M. (2012). O trabalho dos psicólogos nas políticas sociais no Brasil. Avanços em Psicologia Latino-americana, 30 (1), 182-192.

Martins, K. P. H., Matos, T. G. R. \& Maciel, R. H. (2009). Formação em psicologia e as novas demandas sociais: relato dos egressos da Universidade de Fortaleza. Revista Mal-estar e Subjetividade, IX (3), 1023-1042.

Mercadante, O. A., Schechtman, A., Cortes, B. A., Munhoz Junior, E., Mendes, E. V., Un, J. A. W., Medeiros, M., Lemos, M. S. A., Serrano, M. M., Meirelles, R. H. S., Nogueira, R. P., Piola, S., Vianna, S. M. \& Figueiredo, V. C. (2002). Evoluções das Políticas e do Sistema de Saúde no Brasil. In: Mercadante, O. A. et al. (Coord), Caminhos da saúde pública no Brasil (236-313). Rio de Janeiro, RJ. Editora Fiocruz.

Oliveira, I. F. de, Dantas, C. M. B., Costa, A. L. F., Silva, F. L., Alverga, A. R. de, Carvalho, D. B. de \& Yamamoto, O. H. (2004). O Psicólogo nas Unidades Básicas de Saúde: formação acadêmica e prática profissional. Interações, 9 (17), 71-89.

Oliveira, P. R. S. de. (2016). Psicologia e a formação para a saúde: experiências formativas e transformações curriculares em debate. Psicologia: Ciência e Profissão, 36 (2), 280-291.

Pereira, F. M. \& Pereira Neto, A. (2003). O psicólogo no Brasil: notas sobre seu processo de profissionalização. Psicologia em Estudo, 8 (2), 19-27.

Pitombeira, D. F., Xavier, A. S., Barroso, R. E. C. \& Oliveira, P. R. S. de. (2016). Psicologia e a formação para a saúde: experiências formativas e transformações curriculares em debate. Psicologia: Ciência e Profissão, 36 (2), 280-291.

Secretaria De Saúde Do Estado De São Paulo. (2016). Manual de Orientações Técnico Administrativas. Recuperado de: http://www.fmb.unesp.br/Home/ Residencia/manual-de-orientacoes-tecnico-administrativas----versao-maio-2016.pdf.

Seidl, E. M. F \& Costa Junior, A. L. (1999). O psicólogo na rede pública de saúde do Distrito Federal. Psicologia: Teoria e Pesquisa, 15 (1), 27-35.

Yamamoto, O. H. (2006). Graduação e Pós-graduação em psicologia: relações possíveis. Revista Brasileira de Pós-Graduação, 3 (6), 270-281.

Submetido em: 6-12-2017

Aceito em: 18-7-2019 